\title{
Structured Light-based Hazard Detection For Planetary Surface Navigation
}

\author{
Ara Nefian ${ }^{1}$, Uland Y. Wong ${ }^{1}$, Michael Dille ${ }^{1}$, Xavier Bouyssounouse ${ }^{2}$, \\ Laurence Edwards ${ }^{2}$, Vinh To ${ }^{1}$, Matthew Deans ${ }^{2}$ and Terry Fong ${ }^{2}$
}

\begin{abstract}
This paper describes a structured light-based sensor for hazard avoidance in planetary environments. The system presented here can also be used in terrestrial applications constrained by reduced onboard power and computational complexity and low illumination conditions. The sensor consists on a calibrated camera and laser dot projector system. The onboard hazard avoidance system determines the position of the projected dots in the image and through a triangulation process detects potential hazards. The paper presents the design parameters for this sensor and describes the image based solution for hazard avoidance. The system presented here was tested extensively in day and night conditions in Lunar analogue environments. The current system achieves over $97 \%$ detection rate with $1.7 \%$ false alarms over 2000 images.
\end{abstract}

\section{INTRODUCTION}

Planetary rover navigation is constrained by a number of factors including real-time operation requirements, limited onboard computational resources and power, and lack of detailed knowledge of local terrain and illumination conditions. Operations on airless bodies, such as the Moon, Mercury and asteroids, are additionally notable for their extreme imaging conditions of high dynamic range (bright sunlight, dark cast shadows) and backscattering regolith [1]. However, these bodies are also among NASA's highest priority science destinations for sample return and volatiles confirmation.[2].

Autonomous and semi-autonomous rover capabilities are still nascent for airless bodies, particularly in the area of obstacle avoidance. The work presented in this paper describes a structured light "virtual bumper" system for obstacle avoidance in these environments. Virtual bumpers are non-contact, in-close safeguarding sensors that are the last line of defense for hazard detection. Furthermore, due to power considerations, they are typically the only always-on navigation sensors while driving in regions of total darkness (e.g., craters and caves). As such, they must detect obstacles reliably and in real-time within the restrictive requirements of planetary exploration missions.

The virtual bumper system we have developed has been tested in an outdoor test facility under a variety of illumination conditions including bright daylight and night time darkness. While designed specifically for planetary environments, the hazard avoidance system presented here has

\footnotetext{
${ }^{1}$ Stinger Ghaffarian Technologies, Moffett Field, CA, USA \{ara.nefian, uland.wong, michael.dille vinh.to\}@nasa.gov

2 NASA Ames Research Center, Moffett Field, CA, USA \{xavier.bouyssounouse, laurence.j.edwards, matthew.deans, terry.fong $@ @$ nasa.gov
}

terrestrial rover applications including mine and cave exploration and night time traversing. The system uses one or more calibrated laser beam dot projectors and a camera. System design parameters include laser beam intensity, wavelength, orientation and inter-beam angles. A low complexity image processing algorithm detects the projected dots, and based on their location in the image, determines the size of obstacles in the rover's path.

The hazard avoidance system design is described in Section III. The image-based onboard hazard detection algorithm is described in Section IV, and the experimental results are described in section VI.

\section{PRIOR WORK}

Virtual bumper systems have long been employed on mobile robots. Early terrestrial applications utilized noncontact sensing to detect intrusion of personal space in autonomous highway control strategies [3], [4] utilizing RADAR. In modern field robotics, LIDAR virtual bumpers are the preferred method of in-close obstacle detection [5]. Space robotics, in contrast, has not readily adopted timeof-flight technology. Instead, much focus has been placed on structured light triangulation for rovers, and such sensors have been conceived for hazard detection in planetary missions [6] like Mars Pathfinder [7], [8] and for smallsats [9].

Structured light is particularly advantageous in space application due to leveraging of existing cameras and the simplicity of laser diode/diffraction grating hardware. Unlike LIDAR and RADAR, cameras have long legacy of space qualification (radiation, thermal, vacuum) and benefits of low mass and power. Use of active illumination, in addition, has advantages over passive stereo in extreme planetary illumination conditions. The Mars exploration rover, Opportunity, was unable to get acceptable stereo range information from hazard cameras due to lack of texture in the Mars terrain [10]. In dark environments, which are the particular focus of this paper, stereo cannot work without power hungry, active area illumination and is susceptible to dynamic range issues present in imagery from airless bodies.

Specific use of virtual bumpers as a part of a tele-operated robotic system in dark environments was explored in [11]. Three-dimensional, commercial structured light sensing, in the form of Kinect, was utilized in [12] for capability in dark terrestrial rescue environments, but is noted for its poor performance in the presence of direct sunlight. Twodimensional light stripers have been explored for operations 
in direct sunlight, but not with considerations relevant to the Moon [13], [6]. This paper extends prior work to consider structured light virtual bumper technology for lunar surface illumination conditions which straddle the extremes of total darkness and harsh direct sunlight.

\section{Structured Light Design}

The dots projected by the system must be visible under all ambient illumination conditions, including both bright sunlight and total darkness. The conditions on the Moon are considered for this analysis, particularly near the lunar poles to which several potential missions are planned [2]. In this scenario, both brightly sunlit and extremely dark regions shadowed by crater walls or the day-night boundary may appear in the same image.

One operational mode is to repeatedly capture two images from the same location while modulating the projector, one showing the projected dots and the other taken without them. Then, a simple frame differencing-based method can be used to determine the location of the projected dots. However, such a solution, while feasible under many other conditions, would require that the rover stop, which fails to satisfy the requirement of real-time operation. Instead, the method proposed in this paper detects the laser dots from a single image and requires simply that the projected dots outshine all other illumination. This is accomplished by ensuring that the ratio between the pixel intensity due to the laser projector illumination $\left(L_{\text {laser }}\right)$ and the pixel intensity due to ambient illumination $\left(L_{S u n}\right)$ be larger than a fixed threshold $T$. The system design parameterized by laser projector power, wavelength, and operational distance is described by

$$
\left\{\lambda, r, P_{\text {laser }}\right\}=\arg \frac{L_{\text {laser }}+L_{\text {Sun }}}{L_{\text {Sun }}}>T
$$

where $P_{\text {laser }}$ is the projected power over each dot on the ground, $\lambda$ is the laser wavelength, and $r$ is the slant range calculated as the distance between the laser projector and the intersection between the laser beam and surface (Figure 7).

As a simplification of the full light transfer function [14], the pixel intensity due to laser and Sun illumination is modeled by Equations 2 and 3 respectively,

$$
\begin{aligned}
& L_{\text {laser }}\left(\lambda, P_{\text {laser }}\right)=A(\lambda) R\left(\alpha_{l}, i_{l}, e_{l}\right) P_{\text {laser }} C(\lambda) F(\lambda) \\
& L_{S u n}(r)=\int_{\lambda} A(\lambda) R\left(\alpha_{s}, i_{s}, e_{s}\right) I_{s}(\lambda) S(r) C(\lambda) F(\lambda),
\end{aligned}
$$

where $A(\lambda)$ is the lunar albedo at a given wavelength, $R(\alpha, i, e)$ is the Lunar-Lambertian reflectance model chosen here, $I_{s}(\lambda)$ is the wavelength-dependent Sun irradiance, $S(r)$ is the surface area on the ground seen by a pixel in the image at a slant range $r$ from the camera, $C(\lambda)$ is the camera response as a function of the wavelength $\lambda$, and $F(\lambda)$ is wavelength-dependent attenuation due to an optional optical filter placed on the camera.

Figure 1 illustrates the lunar albedo [1] for several lunar terrain regions and a wavelength range between 400$3000 \mathrm{~nm}$. The lunar reflectance model [17] is given by

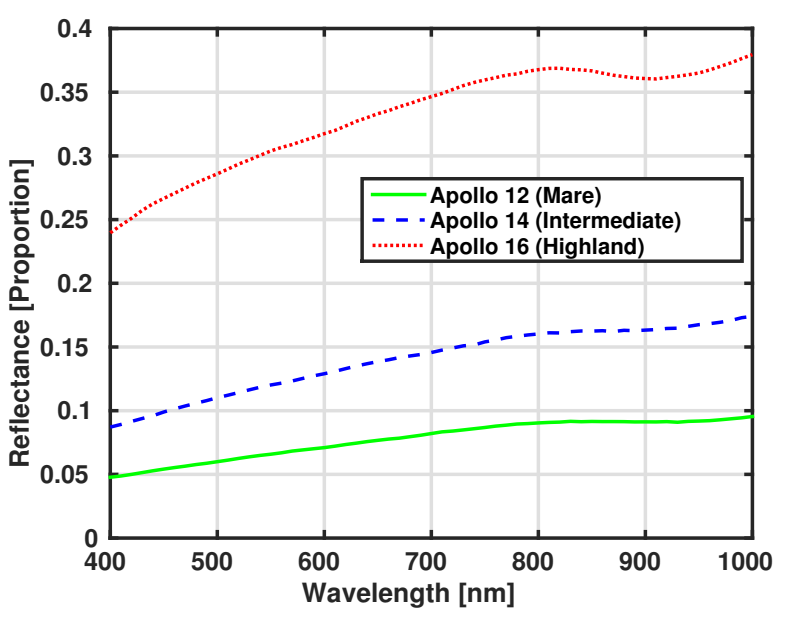

Fig. 1. Albedo for various lunar terrain regions, using soil samples 12001,14163 , and 67641 from [15] and [16].

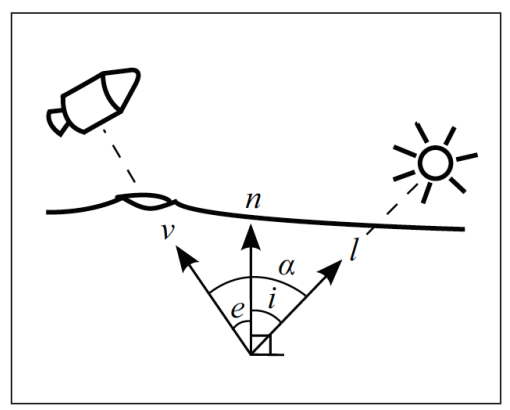

Fig. 2. Reflection angles used in the Lunar-Lambertian model are measured between the view vector (v), lighting vector (l) and the surface normal (n).

Equation 4:

$$
R=R_{0}(\lambda)\left[(1-L(\alpha)) \cos (i)+2 L(\alpha) \frac{\cos (i)}{\cos (i)+\cos (e)}\right]
$$

where $i, e$ and $\alpha$ are the incidence, emission and phase angles illustrated in Figure III respectively. The incidence angle is defined as the angle between the vector of the light source and the surface normal vector at a point on the surface. The emission angle is defined as the angle between normal vector and vector of the camera at the same point on the surface. The angle between the light source vector and camera vector at a point on the surface denotes the phase angle.

The solar irradiance as a function of the wavelength is shown in Figure 3. In a terrestrial application, several atmospheric absorption bands greatly reduce the sun's intensity at many wavelengths, however in the case of the moon lacking any substantial atmosphere, solar irradiance may instead be modeled as originating from a pure blackbody radiator.

Figure 4 illustrates an example camera response function $C(\lambda)$ as a function of the wavelength. An important addition to reduce ambient illumination is the use of an optical filter modeled by $F(\lambda)$ that passes a desirable wavelength band (selected to be centered around the laser projector wavelength (Equation 2) and filters out most of the Sun illumination (Equation 3). For typical filters, $F(\lambda)$ may be modeled 


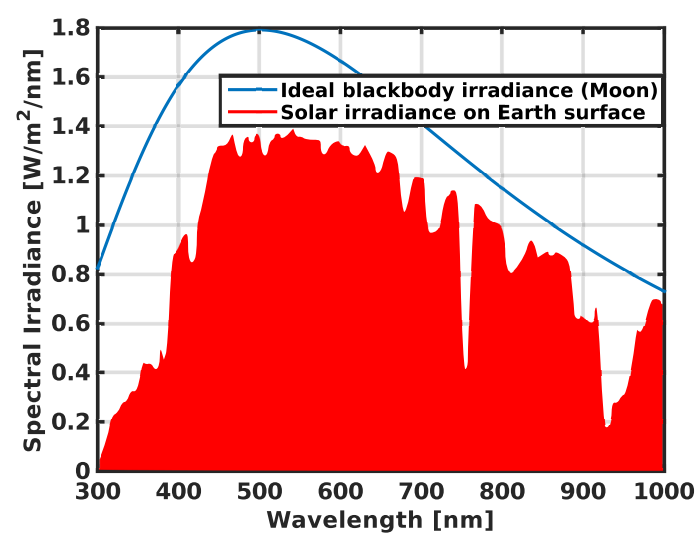

Fig. 3. Solar irradiance on the surface of the earth (with an absorptive atmosphere) and moon (without one). [18]

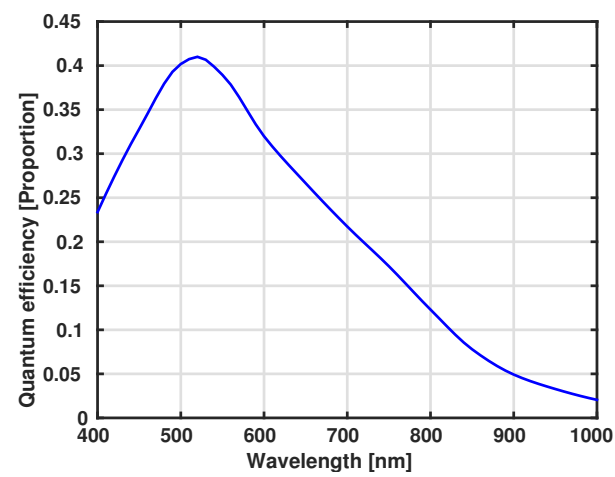

Fig. 4. Camera transfer function for a typical monochrome imager, the Sony ICX267 from the Allied Vision Manta machine vision camera. [19]

as roughly a Gaussian-shaped pass band with a width of approximately $100 \mathrm{~nm}$.

The surface on the ground covered by a pixel in the image, $S$ is approximated by Equation 5 ,

$$
S(r)=\frac{r^{2}}{f^{2}}
$$

where $f$ is the camera focal length in pixels.

Note from Equations 5 and 1 that the shorter the distance $r$ the lower becomes the threshold $T$ making the sensor more robust and more power efficient. However, the distance on the ground between the rover wheels and the obstacle must be larger than the distance required by rover to stop $d_{\text {stop }}$ if an obstacle is detected. The distance $d_{\text {stop }}$ is the distance traveled by the rover during image acquisition, processing and before comes to a complete stop. In a typical configuration the laser projector is oriented at a pitch angle $\theta$ from the rover body as shown in Figure 7. The following equation

$$
r \tan \theta>d_{\text {stop }}
$$

gives a lower bound on the slant range. Equation 6 also shows the importance of a reduced image acquisition and processing time. This constraint plays an important role in the design of the hazard detection algorithm described in Section IV.
A second aspect of the structured light sensor design is to determine its parameters that allow for detection of obstacles of a given height and in a given range $w$ perpendicular to the direction of traverse. These parameters include the pitch angle $\theta$ of the sensor with respect to the rover (Figure 7), the number of laser beams $N$, and inter-beam angle $\gamma$ as illustrated in Figure 5. The laser projector and camera are

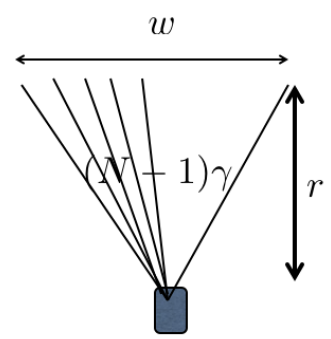

Fig. 5. $\quad N$ laser beams separated by inter-beam angle $\gamma$.

mechanically aligned such that they both have the same roll, pitch, yaw and are separated only by a horizontal baseline, $b$. For a general surface illuminated by the laser projector, the dot positions in the image change compared to the expected dot position on a flat surface. This pixel disparity $\delta d$ relates to the slant range $r$ between the camera and surface along a camera ray as described in the equation below

$$
\delta r=\frac{r^{2}}{b f} \delta d
$$

where $f$ is the camera focal length (in meters). The triangulation process is illustrated in Figure 6. If the camera and

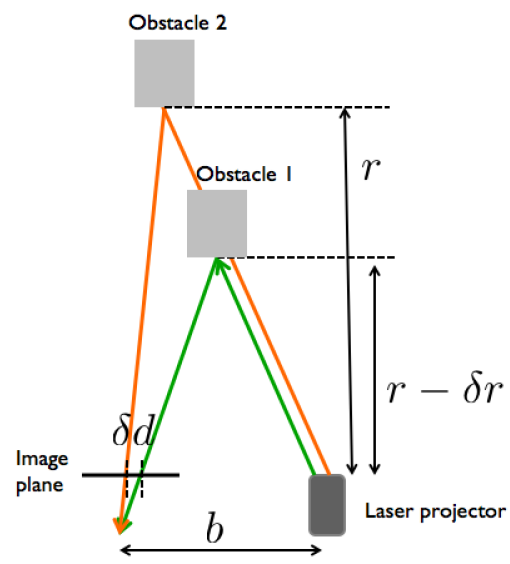

Fig. 6. The projector and camera triangulation process. Obstacles at distance $r$ and $r-\delta r$ appear in the image separated by a pixel disparity $\delta d$.

laser projector system are looking at the surface under a pitch angle $\theta$, (Figure 7) the variation in obstacle height $\delta h$ that corresponds to a pixel disparity $\delta d$ is given by

$$
\delta h=\delta r \cos (\theta)=\frac{r^{2}}{b f} \delta d \cos (\theta)
$$

The above equation defines the minimum pixel disparity to detect obstacle higher than a given $\delta h$. The number of 


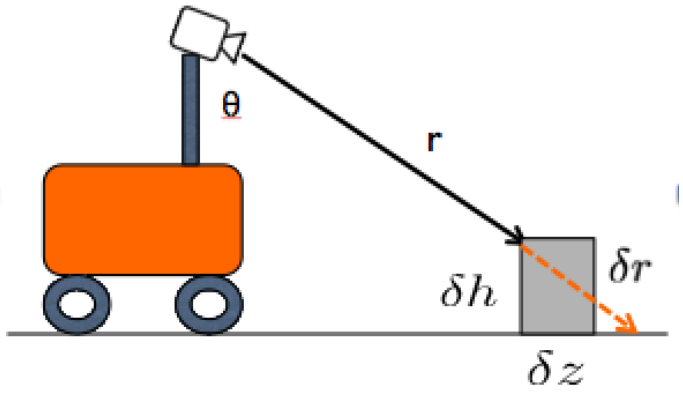

Fig. 7. Distance from laser projector mounted on the rover to ground obstacles.

laser beams $N$ to cover a segment $w$ perpendicular to the rover traverse at a distance $r \sin (\theta)$ in front of the rover is calculated as

$$
N=\frac{2}{\gamma} \tan ^{-1}\left(\frac{\frac{w}{2}}{r}\right)+1
$$

If the number of laser beams cannot be achieved with single laser projector, a solution with multiple projectors is chosen to satisfy Equation 9. The number of beams must also satisfy the condition

$$
\frac{w}{N}>\delta d
$$

If the above condition is not satisfied the system will detect as obstacles terrain features that are smaller than $\delta h$ and generate a large number of false alarms.

\section{HAZARD DETECTION}

The hazard avoidance system uses the laser dots projected in the camera to determine potential obstacles in the rover path. In a calibrated system the camera and laser dot projector have the same orientation and pose except a horizontal baseline $b$ as described in Section III. For this system all projected dots align on the same epipolar line. The obstacles in front of the camera will determine only a shift in the horizontal position of the laser illuminated pixels. Their vertical position remains on the same image row. This solution reduces significantly the image acquisition and processing time since the search for the laser dot location reduces to a single image line. It also relaxes the condition in Equation 6 by reducing the distance $d_{\text {stop }}$ needed by the rover to stop in front of an obstacle. Let ROW_INDEX be the image row where all laser dots are projected and let LEFT_COL and RIGHT_COL be the columns corresponding to the left most and right most laser beams projected on the image on a flat surface. An image region of interest (ROI) is defined by the following boundaries. The top left corner of the ROI is (LEFT_COL- $\delta d$, ROW_INDEX-D) and right bottom corner is (RIGHT_COL+ $\delta d, \mathrm{ROW}$ INDEX+D), where $\mathrm{D}$ is a fixed number of image rows. $\delta d$ is computed using Equation 8 for the given minimum obstacle height $\delta h$, and given sensor pitch $\theta$. Note that with this choice of $\delta d$ and ROI boundaries and in the presence of obstacles of height larger than $\delta h$, the number of dots projected within the ROI is lower than the number of laser beams $N$.
Figure 8 describes the overall system for hazard avoidance using the structured light sensor.

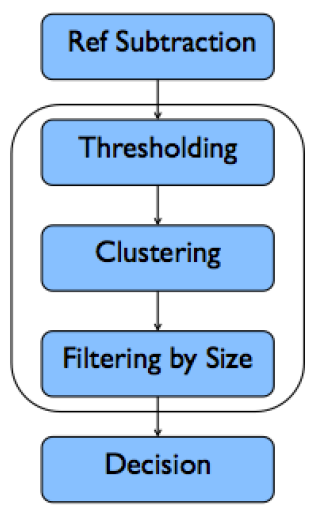

Fig. 8. Overall system

\section{Reference Subtraction and Thresholding}

When there are two images available captured with and without the laser projected dots from the same camera pose and same illumination conditions, the image difference between these two images will select primarily the pixels illuminated by the laser together with low intensity image noise. This solution requires the rover to stop and insure the two images are captured from the same camera pose. The threshold value is selected such that $\frac{L_{\text {laser }}}{L_{\text {noise }}}>T$. Using a single image showing the projected laser dots allows to select all pixel illuminated by the laser projector using a fixed threshold value $T$ such that $\frac{L_{\text {laser }}}{L_{\text {Sun }}}>T$. In this case the laser projected power must be significantly higher to insure that terrain features of various slopes and albedo values are not confused for projected laser beams (Equation 1). Alternatively, under the assumptions of slow varying terrain features, a "background" ROI can be used. The background ROI has the same width, height and left horizontal pixel value as the ROI containing the projected laser dots. It is only shifted vertically by a fixed number of pixels. The "background"ROI approximates the appearance of the background image when the background image is not available and the threshold value $T$ in this case is selected such that $\frac{L_{\text {laser }}}{L_{\text {noise }}}>T$. This solution allows for continuous rover operation and for reduced laser power intensity but relies on the assumption of a slow varying terrain features. The output of this processing stage is a binary image of the ROI size obtained through thresholding. Pixels with intensity values above $T$ value are set to one. All other pixels are set to zero and discarded.

\section{Clustering and Filtering by Size}

The pixels in the binary image are grouped into several clusters using a connected component algorithm. The centroid of each cluster is computed through averaging of the position of all pixels belonging the the cluster. Clusters with a number of pixels below a fixed threshold are removed.

\section{Obstacle Decision}

The detection algorithm determines the number of laser dots within the ROI and their relative position. When the number 
of detected centroids in the ROI is lower than the number of laser beams $N$, the algorithm detects an obstacle. This is due to the size of the image ROI relative to the maximum pixel disparity for a given acceptable obstacle. This case corresponds to occlusions or the detections of wide obstacles (positive or negative) or terrain slopes that shift all laser dots horizontally by more than $\delta d$.

If the number of detected dots equals the number of laser beams $N$, a simple correspondence based on pixel order is established between the expected location of the dots on a flat surface and their detected location. If the (horizontal) distance between the expected and detected dot position is larger than $\delta d$ for any dot, the algorithm detects an obstacle. This case corresponds to narrow obstacles (positives or negatives) that do not influence the location of all dots but only a subset of them.

\section{EXPERIMENTAL SETUP}

A virtual bumper prototype was developed for testing and is shown in Figure 9 (right). The system utilizes a commercial machine vision camera [19] and a commercially available red light $(660 \mathrm{~nm})$ laser projector with $11 \mathrm{dot}$ diffraction grating [20]. The baseline between camera and laser projector is set to $26 \mathrm{~cm}$ and the pitch angle $\theta=28 \mathrm{deg}$.

Physical experimentation was performed with the virtual bumper on NASAs K-REX2 rover (Figure 9). The goals were to stress the design and learn about the parameters and performance in a Lunar-relevant environment. Testing occurred at NASA Ames Research Centerss Roverscape facility, a two-acre outdoor planetary analog terrain with boulder distributions and average surface albedo (8\%) similar to Lunar regolith. However, the free surface is covered in a layer of pea gravel and not a regolith (moon dust) simulant for reasons of practicality. Day and night tests were conducted to recreate the illumination conditions in direct sunlight and inside shadowed craters, respectively. Though daytime illumination distribution and spectrum are not exact standins for the moon, which lacks an atmosphere, clear sky days were leveraged such that total incident illumination and dominant directionality were similar.
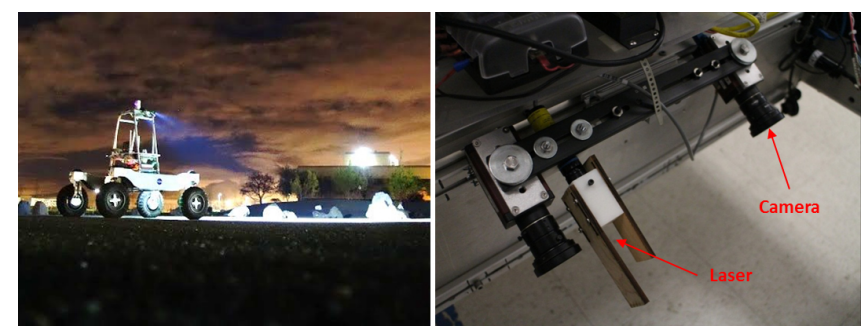

Fig. 9. Experimentation utilized NASAs K-REX2 rover, pictured here during night testing in the Roverscape (left). The virtual bumper experimental setup comprises a multi-dot laser projector and camera mounted on the leading edge of the rover (right).

Obstacle test cases were designed to mirror the geometric possibilities likely to be seen in craters at the poles of the moon. Figure 10 shows examples of the four main types of the obstacles encountered along with their relative sizes and reflectivities: large angular rocks characteristic of the polar surface areas, ejecta blocks characteristic of craters, smaller angular rocks, and complex aggregates from multiple small obstacles. Obstacle albedos ranged from $<10 \%$ to $33 \%$, and were dissimilar to the general terrain surface albedo. This presents a way of testing incident faces on obstacles that would be free of regolith accumulation. Within the scope of this paper, all of the obstacles tested are positive (rocks) which reflects the relative prevalence of rocks versus craters and gaps (negative) in the size danger zone.

The K-REX2 rover was manually driven in the Roverscape while pushbrooming the virtual bumper out front. Contact with obstacles was always made in the direction of pushbrooming that is, no point turn obstacles. A variety of speeds between 0 and $20 \mathrm{~cm} / \mathrm{s}$ were tested with $10 \mathrm{~cm} / \mathrm{s}$ being the most common. Manual driving enabled the rover to weave cleanly between and reverse from obstacles without biasing results with navigation software in the loop. Images were taken at $0.5 \mathrm{~Hz}$ rate, reflecting a mission requirement, timestamped, and processed offline. Obstacles were hand labeled in images with guidance from flagged timestamps where obstacles were encountered. The camera was locked
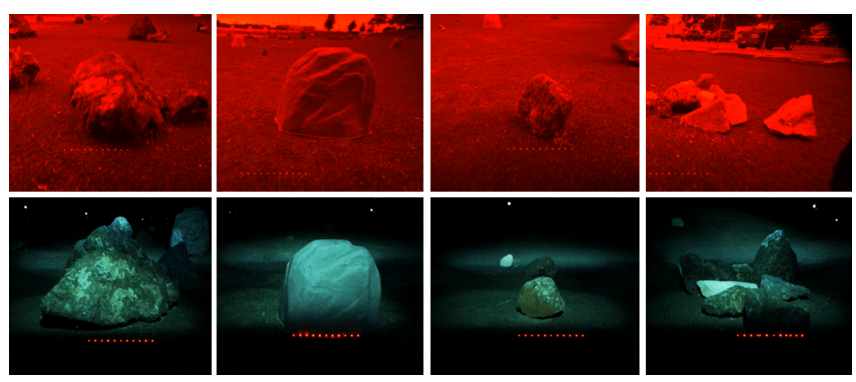

Fig. 10. Rock types used in day and night testing included (left to right) large angular rocks, ejecta blocks, small angular rocks, and complex multirock aggregates.

at F/5.6 aperture, focal length $3.5 \mathrm{~mm}$, and ISO400 equivalent gain, while independent shutter speeds were chosen for day and night testing. These fixed shutter values were hand-tuned to produce $90 \%$ saturation of the laser dots in the resulting image (i.e. $0.9 \times 255=230$ for 8 -bit images). Ideally, a single shutter speed would be used for all conditions, thus enabling use of the system across bright scenes with dark cast shadows. However, due to dynamic range limitations of the commercial machine vision camera, it was decided to collect clean data instead of introducing a minor operational detail. Other optical settings were chosen to reflect good depth of field and low pixel noises.

An image threshold controls what brightness level to accept if a pixel is illuminated by a laser dot (Figure 11). Two optimal thresholds exist for night and day due to the differences in total exposure. The large spread between these empirical values ( $50 \%$ ) stems from both the decision to use different shutter speeds and the change in ambient illumination. We believe that while two optimal thresholds will exist due to the latter reason, the much smaller spread 
will allow a single threshold to work well in night and day images.

The minimum pixel disparity threshold $\delta d$ determines if each laser dot has detected an obstacle based on the distance between the dot and its ideal flat-groundplane location along the epipolar line as discussed in Equation 8. The analytical value of $\delta d$ for our system parameter and obstacles of height $h=10 \mathrm{~cm}$ is 15.9 pixels. As the system is designed with a neutral spacing of 20 pixels between laser dots, there is an upper disparity limit at which it is impossible to identify a shifted dot between neighbors (and granular obstacle detection capability is lost), though the software allows for such values (Equation 10). Based on validation data, it was empirically determined that a threshold of 15 ( $75 \%$ of the ideal spacing) strikes the optimal balance of precision and sensitivity (Figure 12).

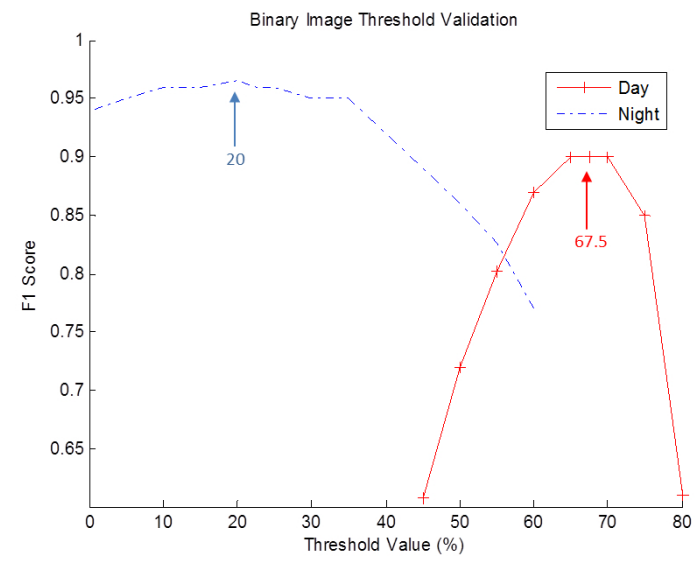

Fig. 11. Selection of binary image threshold values using F1 score as a metric. Spread of data between day and night are due to natural changes in ambient illumination and artifical changes in camera settings.

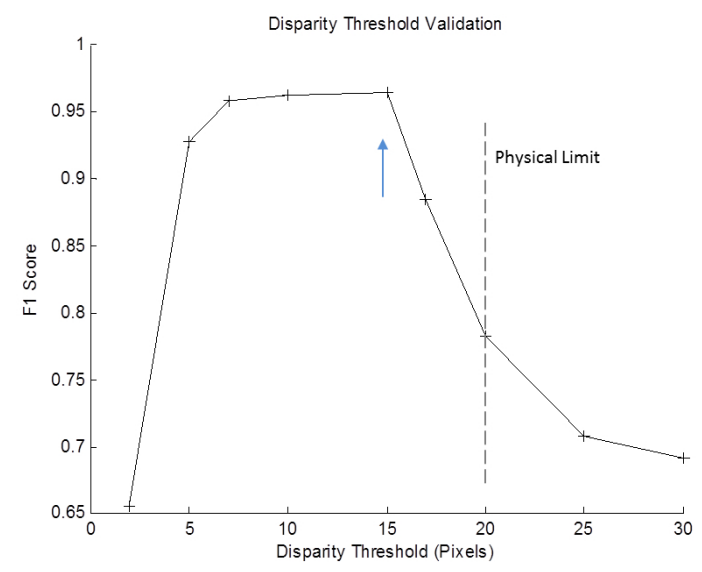

Fig. 12. Validation of the minimum disparity threshold parameter for detecting obstacles on experimental data. The value found empirically (15 pixels) is close to the analytic ideal of 15.9 pixels.

\section{RESUlTS AND Discussion}

Results from testing the virtual bumper on the Roverscape are shown in Figure 13. 1158 day images across two tests and 1544 night images were used in evaluation of the virtual bumper. Between $30-40 \%$ of these images were of obstacles and the rest were from clear paths. Two daylight tests were run with mechanical configurations that optimized detection for different target distances. Tests utilizing the second, $0.7 \mathrm{~m}$ configuration were conducted when it was noticed that the apparent brightness of the laser dots over the background terrain was much less than anticipated (see discussion of errors below). The closer target distance increased the perceived brightness of the laser dots due to the reduction in square distance illumination falloff and a change in incident angle on the terrain towards the perpendicular.

The positive obstacle detection accuracy of virtual bumper is around $97 \%$, and is not significantly different between the day and night images. However, the false alarm rate (detection of obstacle when actually clear) varies significantly from $1.7 \%$ at night to $4.1 \%$ in the worst of the day tests. This concurred with the visual observation that dots were challenging to perceive in daylight, despite use of monochromatic laser and narrow band filter. The average F1 score for day images is 0.92 compared to 0.96 for night.

\begin{tabular}{|c|c|c|c|}
\hline \multirow[b]{2}{*}{ Target Distance } & \multicolumn{2}{|c|}{ Day } & \multirow{2}{*}{$\begin{array}{l}\text { Night } \\
1.2 \mathrm{~m}\end{array}$} \\
\hline & $0.7 \mathrm{~m}$ & $1.2 \mathrm{~m}$ & \\
\hline Total Images (\#) & 529 & 629 & 1544 \\
\hline \# Obstacles & 119 & 147 & 444 \\
\hline \# Clear Path & 410 & 482 & 1100 \\
\hline True Pos & 117 & 139 & 431 \\
\hline True Neg & 395 & 462 & 1081 \\
\hline False Pos & 15 & 20 & 19 \\
\hline False Neg & 2 & 8 & 13 \\
\hline Obstacle Acc (\%) & 98 & 95 & 97 \\
\hline False Alarm Rate (\%) & 3.6 & 4.1 & 1.7 \\
\hline F1 Score & 0.93 & 0.91 & 0.96 \\
\hline Total Day Images & \multicolumn{2}{|c|}{1158} & \\
\hline Avg Day Fl & \multicolumn{2}{|c|}{0.92} & \\
\hline
\end{tabular}

Fig. 13. Table of Results

Images representing typical erroneous classification in day and night testing are shown in Figure 14. In day and night images, the most common false positive errors were the dots speckling as the rover drove over clear terrain or the introduction of new dots in the region of interest by bright terrain spots (day only). The major source of these errors is the result of terrestrial factors not represented in the ideal reflectance model. These factors included the blanket of granular pea gravel, compared to fine, powdered regolith which covers the moon. These granules both act as tiny occluders and introduce variegation in the terrain as they are not made of a macroscopically uniform material. The total effect was such that the variability in surface terrain reflectance was at times greater than the contribution of the laser illumination, causing dots to speckle in the imagery. In 
day and night images, many false negative errors stemmed from labeling at the transition between clear ground terrain and rock obstacles. These labels have great uncertainty and given possibilities to exploit the sequential nature of pushbroom images, great performance in these regions is not important. Upon discarding these transition images, the positive obstacle accuracy is over $99 \%$. A source of false negative error specific to night images is smearing of dots due to motion blur from long exposure time. While a dot that has moved sufficiently along the epipolar line should trigger a detection, motion blur creates an elongated blob where the centroid is within the disparity tolerance. This has reinforced the importance of fast shutter speeds, though it was unavoidable at the time due to camera availability.

It can be argued that the most important purpose of the system to is correctly identifying obstacles which would cause catastrophic failure. In this, virtual bumper has performed quite well. Moreover, virtual bumper is perhaps the last line of defense in dark craters, where a Lunar rover will be spending most of its time with intermittent stereo and flash illumination. The strong dark performance demonstrated here highlights virtual bumpers value for this niche purpose. Thus, these results are promising for early proof-of-concept. However, in future development, more realistic reflectance environments will be emphasized and with laser technology with greater power output will be investigated.
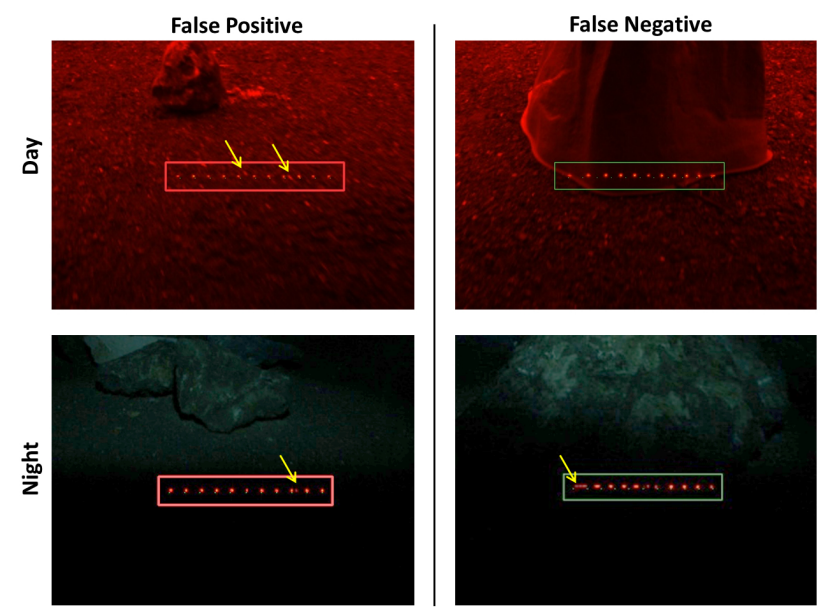

Fig. 14. Error examples from day and night testing. False positives result from variegation of terrain. False negatives result from uncertain labeling of transition regions between obstacle and clear ground. Motion blur also caused false negatives in night images.

\section{CONCLUSIONS}

This paper demonstrates the usability of a structured light sensor for hazard avoidance in planetary navigation as well as for terrestrial applications restricted by low onboard power and low computational complexity. The sensor design methodology for a given set of constraints is discussed and a low complexity image-based hazard avoidance method is presented. The method has been tested in several day and night scenarios in a Lunar analog environment. The current system achieves over $97 \%$ detection rate with $1.7 \%$ false alarms. Future work will be directed towards the use of higher power laser projectors. The capabilities of the image based hazard avoidance method will be enhanced to handle robustly high reflectance surface regions and multiple reflections from the same projected beam.

\section{REFERENCES}

[1] D. McKay, H. Grant, A. Basu, G. Blanford, et al., "The lunar regolith," in Lunar Sourcebook: a user's guide to the moon.

[2] National Research Council : Committee on the Planetary Science Decadal Survey, "Vision and voyages for planetary science in the decade 2013-2022,"

[3] B. Schiller, V. Morellas, and M. Donath, "Collision avoidance for highway vehicles using the virtual bumper controller," in Proceedings IEEE International Conference on Intelligent Vehicles.

[4] M. Hennessey, C. Shankwitz, and M. Donath, "Sensor based "virtual bumpers" for collision avoidance: Configuration issues," in Proc. SPIE 2592, Collision Avoidance and Automated Traffic Management Sensors.

[5] C. Urmson, M. Dias, and R. Simmons, "Stereo vision based navigation for sun-synchronous exploration," in Proceedings IEEE/RSJ Conference on Intelligent Robotics and Systems.

[6] Larry Matthies, Tucker Balch, and Brian Wilcox, "Fast optical hazard detection for planetary rovers using multiple spot laser triangulation," in Robotics and Automation, 1997. Proceedings., 1997 IEEE International Conference on. IEEE, 1997, vol. 1, pp. 859-866.

[7] H. W. Stone, "Mars pathfinder microrover: A low-cost, low-power spacecraft," in Forum on Advanced Developments in Space Robotics Robotics and Automation. Proceedings. AIAA, 1996.

[8] L. Matthies, Erann. Gat, R. Harrison, et al., "Mars microrover navigation: Performance evaluation and enhancement," in Proceedings IEEE/RSJ International Conference on Intelligent Robots and Systems, 1995.

[9] C. Liebe, C. Padgett, J. Chapsky, D. Wilson, et al., "Spacecraft hazard avoidance utilizing structured light," in Proceedings IEEE Aerospace Conference.

[10] M. Maimone, A. Johnson, Y. Cheng, R. Willson, and L. Matthies, "Autonomous navigation results from the mars exploration rover (mer) mission," in Proceedings 9th International Symposium on Experimental Robotics, 2006.

[11] K. Kon, Y. Urano, N. Shiroma, Sato N., et al., "Development of robot teleoperation system in bad viewing condition," in Proceedings IEEE International Conference on Robotics and Biomimetics.

[12] J. Suarez and R. Murphy, "Using the kinect for search and rescue robotics," in IEEE Symposium on Safety, Security and Rescue Robotics.

[13] C. Mertz, J. Kozar, J. Miller, and C. Thorpe, "Eye-safe laser line striper for outside use," in Intelligent Vehicle Symposium.

[14] BKP Horn, "Exact reproduction of colored images," in Computer Vision, Graphics and Image Processing 26:135-167, 1984.

[15] C.M. Pieters, L. A. Taylor, D. S. McKay, S. Wentworth, R. V. Morris, and L. P. Keller, "Spectral characterization of lunar mare soils," in Lunar and Planetary Science Conference XXXI.

[16] L. A. Taylor, A. Patchen, J. Cahill1, C.M. Pieters, R.V. Morris, L.P. Keller, and D.S. Mckay, "Mineral and glass characterization of Apollo 14 soils," in Lunar and Planetary Science Conference XXXIII.

[17] A. S. McEwen, "Photometric functions for photoclinometry and other applications," in Icarus, 92:298-311.

[18] K. N. Liou, An Introduction to Atmospheric Radiation, Academic Press, 2002.

[19] Allied Vision Technologies, "Gige vision camera with Sony ICX267 and PoE option," in http://www.alliedvisiontec.com/emea/products/cameras/gigabitethernet/manta/g-146bc.html.

[20] Osela Inc., "Osela Streamline Laser," in http://www.osela.com/wpcontent/uploads/2015/08/OSELA_DATA_SHEET_STREAMLINE_2015 .$p d f$. 